\title{
LUCIFERASE REPORTER MYCOBACTERIOPHAGES FOR EVALUATING NORBORNENE-BASED ANTITUBERCULOSIS DRUG SUSCEPTIBILITY TESTING ON MYCOBACTERIUM TUBERCULOSIS
}

\author{
KALAISELVI $\mathrm{K}^{1}$, MANGAYARKARASI ${ }^{1 *}$, GOMATHI NS ${ }^{2}$, BALAJI ${ }^{2}$, SHIVSHANKAR R MANE ${ }^{3}$, RAJA SHUNMUGAM ${ }^{3}$ \\ ${ }^{1}$ Department of Microbiology, SRM Medical College Hospital and Research Centre, Kattankulathur, Kanchipuram, Tamil Nadu, India. \\ ${ }^{2}$ Department of Bacteriology, National Institute for Research in Tuberculosis, Chetpet, Chennai, Tamil Nadu, India. ${ }^{3}$ Department of \\ Chemical Sciences, Polymer Research Centre, Indian Institute of Science Education and Research, Kolkata, West Bengal, India. \\ Email: mangayarkarasi.v@ktr.srmuniv.ac.in
}

Received: 04 May 2017, Revised and Accepted: 19 July 2017

\section{ABSTRACT}

Objective: In 2015, 9.6 million people around the world became sick with tuberculosis (TB) disease and 1.5 million TB-related deaths worldwide. Recent increasing incidence of multidrug-resistant (MDR; resistance to at least rifampicin (RIF) and isoniazid [INH]) and extensively drug-resistant (MDR resistance plus resistance to a fluoroquinolone and an aminoglycoside) makes TB a serious concern. Lot of research is needed to deal with this infectious disease for a better alternative in treatment or modification of these older TB drugs. The present study aimed at evaluating antimycobacterial activity of norbornene (NOR) derived INH copolymer with poly ethylene glycol (NOR- polyethylene glycol [PEG]-INH) a novel nanocarrier along with the anti-TB drug using luciferase reporter phages (LRPs).

Methods: NOR derived INH accounts for $74 \%$ of INH, $24 \%$ of NOR, and $2 \%$ of PEG. H37Rv control strain, a sensitive, and a resistant strain of Mycobacterium TB (MTB) used in this study. The in vitro activity of the drug was evaluated using absolute concentration method. The resistant strain was evaluated using LRP assay to observe the minimum inhibitory concentration of the drug.

Results: Reduction in light units was observed for the resistant strain exposed to plain INH and NOR-PEG-INH separately. $24 \%$ of reduction was observed in strains exposed to plain INH whereas $37 \%$ of reduction was observed in strains exposed to NOR-PEG-INH.

Conclusion: NOR-based INH had better antimycobacterial activity compared to plain INH and RIF. Antimycobacterial activity of INH and RIF increases even with very low dosage with NOR conjugate.

Keywords: Norbornene, Antimycobacterial activity, Minimum inhibitory concentration, Mycobacterium tuberculosis.

(C) 2017 The Authors. Published by Innovare Academic Sciences Pvt Ltd. This is an open access article under the CC BY license (http://creativecommons. org/licenses/by/4. 0/) DOI: http://dx.doi.org/10.22159/ajpcr.2017.v10i9.19660

\section{INTRODUCTION}

Tuberculosis (TB) is considered as the deadly disease of the past and present in the earth [1]. The current TB chemotherapy under directly observed therapy short course even though highly efficient, control of TB is highly challenging [2]. Long duration and complexity of the chemotherapy results in non-adherence to the treatment leading to crucial response such as failure or relapse or multidrug-resistance TB and extensively drug-resistant TB in patients [3]. Newer anti-TB drugs, namely, delamanid, bedaquiline, and pretomanid has a good synergistic effect but attempts to shorten the course of treatment with these alternative drugs resulted in severe adverse effects $[4,5]$.

At present, there is a pressing need to improve treatment by either enhancing the application of existing agents with nanocarriers or introduction of the new drugs which shorten the treatment. The drug delivery systems have produced novel solutions to reduce the side effects and duration of treatment using these four drugs through nanotechnology [6].

Nanotechnology is a technology which uses materials of nanometer scale length (1-100 nm) can be used for a broad range of applications and the creation of various types of nanomaterials and nanodevices [7]. Nanotechnology has provided a huge development to pharmacology through the designing of drug delivery systems. Polymers act as a nanocariers for drug delivery $[8,9]$. Norbornene (NOR) derived doxorubicin polymeric micelle with hydrazone linker shows excellent performance inside the cell as an efficient drug delivery system and longer bioavailability. NOR derived thiobarbiturate homopolymeric symbolize themselves as promising carriers for the stimuli triggered intracellular delivery of hydrophobic drugs [10-12].

NOR derived anti-TB drugs can be a multi frontline TB drugs for potential therapeutic treatment. It has stability under physiological condition and depending on acidic $\mathrm{pH}$ condition the drug releases and has the feasibility of potential drug delivery in macrophage compartments [13]. The interaction of NOR along with isoniazid (INH) toward Mycobacterium TB (MTB) has to be studied detailed with drug susceptibility testing (DST). Various phenotypic methods are utilized for DST of MTB including absolute concentration method (ACM), proportion method, resistance ratio method, alamlar blue assay, and luciferase reporter mycobacteriophage (MP) assay [14].

Luciferase reporter phage (LRP) assay involves viable mycobacteria infected with reporter phages (phAETRC21) expressing firefly luciferase gene. Light production requires metabolically active MTB cells, in which reporter phages replicate and luciferase gene will be expressed. Drug-susceptible MTB strains with specific anti-TB drugs, fail to produce light after infection with LRPs but drug-resistant strains are unaffected by the drugs and produce light similar to the strains which are not exposed to drug (control) [15]. The aim of the study was to evaluate the efficacy of NOR anti-TB drugs susceptibility testing on MTB using Luciferase reporter MP. 


\section{METHODS}

The plain nanocarrier with INH was procured from Department of Polymer Chemistry, IISER, Kolkata, India. INH attached to the NOR monomers through a stimuli responsive linker by covalent binding method. NOR covalent binding with INH is shown in Fig. 1.

H37Rv strain, of MTB received from National institute for Research in TB (NIRT) was used as the standard strain. The Institute Ethical Committee approval was obtained and informed consent were received from the TB patients. A sensitive strain and resistant strain were identified from the sputum received from the patients. $\mathrm{H} 37 \mathrm{Rv}$, sensitive strain and resistant strain were grown in Lowenstein Jensen (LJ) medium.

\section{Drug preparation}

As per the ICMR MANUAL $20 \mathrm{mg}$ of NOR-polyethylene glycol [PEG]INH was dissolved in $20 \mathrm{ml}$ of sterile distilled water to make up $10,000 \mu \mathrm{g} / \mathrm{ml}$ which serves as a stock solution, from the stock solution working solution was prepared by further dilution. The stock solution was maintained at $-20^{\circ} \mathrm{C}$, working solution was used for the incorporation of the drug into LJ's medium.

\section{ACM}

To calculate efficacy of the drugs, DST was performed using ACM. The following concentration of anti-TB nanodrugs was used in this study; $0.025,0.05,0.1,0.2,0.5,1,2.5$, and $5 \mu \mathrm{g} / \mathrm{ml}$ for INH. Drug-free media and media containing graded concentration of the drugs were inoculated with the standard strain H37Rv of MTB, sensitive strain and a resistant strain and readings were taken on $28^{\text {th }}$ day [14].

\section{Phage propagation (MP)}

MP buffer was prepared. $500 \mu \mathrm{l}$ of phage buffer was added in cryovial. $50 \mu \mathrm{l}$ of the phage was added in the first vial and $50 \mu \mathrm{l}$ to the next. The phages were diluted up to the desired dilution in the same buffer. $500 \mu \mathrm{l}$ of bacterial suspension (Mycobacterium smegmatis) equivalent to 2-3 McFarlands standard to the last three dilutions were added and incubated at $37^{\circ} \mathrm{C}$ for 30 minutes. After incubation, $200 \mu$ from each dilution was taken and mixed with $5 \mathrm{ml}$ of soft agar separately and poured on to the $7 \mathrm{H} 9$ base medium, the plates were incubated at $37^{\circ} \mathrm{C}$ for $18-24 \mathrm{hrs}$. The plates showing lacey pattern of plaque formation was chosen to harvest the phages. $5 \mathrm{ml}$ of MP buffer was added to the plates, shaken for $2 \mathrm{hrs}$ at room temperature in rotatory shaker $(\sim 80 \mathrm{RPM})$. The buffer was aspirated and filtered using $0.45 \mu \mathrm{m}$ membrane filter. The dilution was marked in which phages harvested with date and stored at $4^{\circ} \mathrm{C}[15]$.

\section{LRP DST}

From the culture suspension prepared for DST LRP (DLRP), $100 \mu$ was transferredto 4 sterile cryovials. $400 \mu \mathrm{l}$ of 7H9 medium was added to all the four vials (Two vials serves as blank and third vial for INH and fourth vial for NOR+PEG+INH). Mix and incubate at $37^{\circ} \mathrm{C}$ for $72 \mathrm{hrs} .50 \mu \mathrm{l}$ of phAETRC21 and $20 \mu \mathrm{l}$ of $0.1 \mathrm{M} \mathrm{CaCl}_{2}$ were added to all vials. Incubated at $37^{\circ} \mathrm{C}$ for $4 \mathrm{hrs} .100 \mu \mathrm{l}$ of the cell-phage mixture was transferred to a luminometer cuvette. $100 \mu \mathrm{l}$ of D-Luciferin was added and reduction in light units (RLU) was immediately measured at 10 seconds integration. Luminometer cuvette was discarded in to the lysol bath [16].

The percentage reduction in RLU was calculated using:

$$
\% \text { Reduction in RLU }=\frac{\text { Control RLU }- \text { Test RLU }}{\text { Control RLU }} \times 100
$$

\section{RESULTS AND DISCUSSION}

The nanocarriers with anti-TB drugs were validated using scanning electron microscope and atomic force microscopy for their size in IISER Kolkata [13]. INH was dissolved in distilled water.

Minimum inhibitory concentration (MIC) of three strains with plain TB drugs and NOR based TB drugs showed that NOR+PE+-INH showed anti-TB activity with lower concentration. The LJ medium bottles used for ACM were shown in Fig. 2. The significant antimycobacterial activity of $\mathrm{NOR}+\mathrm{PEG}+\mathrm{INH}$ of the sensitive strains and resistant strains in comparison with INH were explained in Table 1. NOR based nanaocarriers have a capacity to interact with antituberculous drugs and form a copolymer. The presence of antimycobacterial activity of $\mathrm{NOR}+\mathrm{PEG}+\mathrm{INH}$ against MTB indicates that the formed copolymer does not affect the original mechanism of anti-TB activity of the drug INH. The copolymer and the anti-TB drugs directly interact with MTB the results were similar to that of another study done using analogue of INH [16].

In ACM NOR+PEG+INH on resistant strain, no inhibitory action was observed. INH losses their activity while binding to egg proteins, which are the ingredients of LJ medium [17]. Alternative DST method of MP with luciferin was used in this study. The plate which is used for harvesting phages was shown in Fig. 3. LRPs has various advantages such as less time-consuming and do not involve any tedious

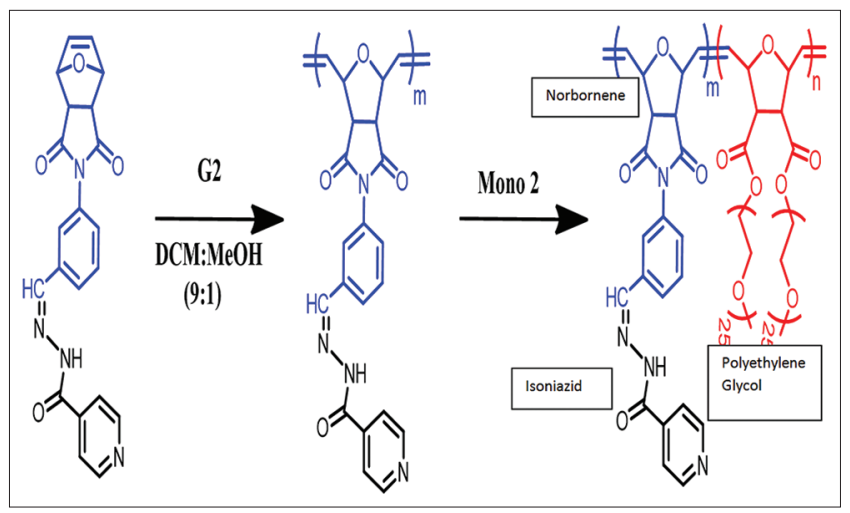

Fig. 1: Chemical structure of norbornene linked with isoniazid

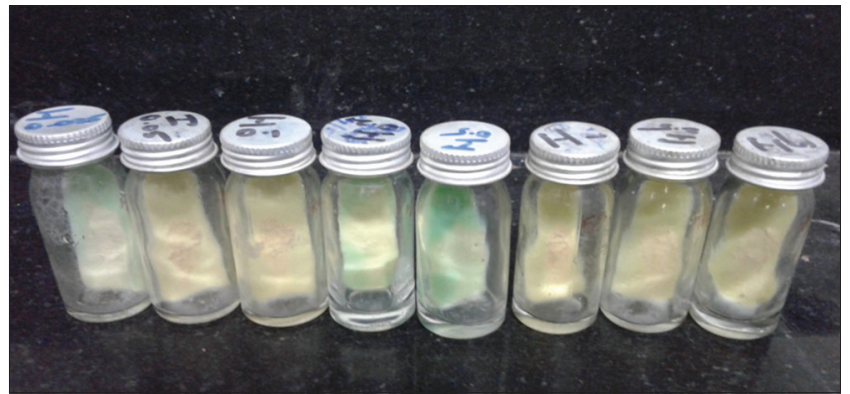

Fig. 2: Drug susceptibility testing performed in Lowenstein Jensen medium using absolute concentration method

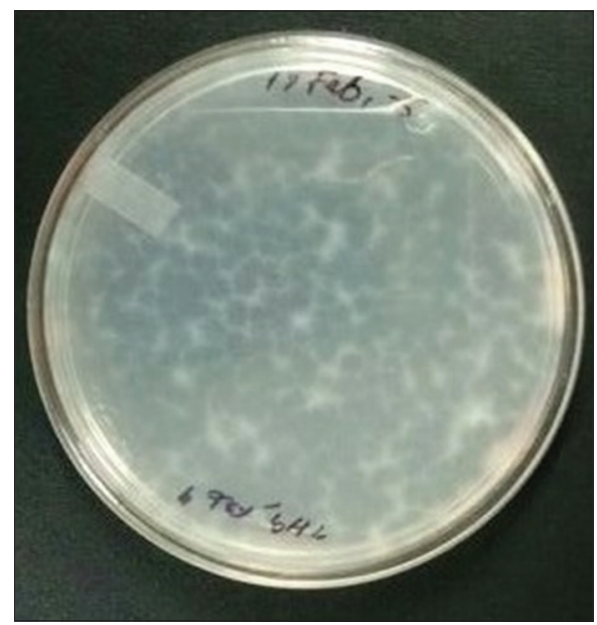

Fig. 3: Plate showing lacey pattern 
Table 1: MIC of plain and NOR conjugate with INH by ACM

\begin{tabular}{lll}
\hline \multirow{2}{*}{ Control and clinical strains } & \multicolumn{2}{l}{ MIC of Anti-TB drugs } \\
\cline { 2 - 3 } & Plain INH & NOR+PEG+INH \\
\hline H37Rv & $0.025 \mu \mathrm{g} / \mathrm{ml}$ & $1 \mu \mathrm{g} / \mathrm{ml}$ \\
Sensitive strain & $0.5 \mu \mathrm{g} / \mathrm{ml}$ & $1 \mu \mathrm{g} / \mathrm{ml}$ \\
Resistant strain & $2.5 \mu \mathrm{g} / \mathrm{ml}$ & $5 \mu \mathrm{g} / \mathrm{ml}$ \\
\hline
\end{tabular}

TB: Tuberculosis, MIC: Minimum inhibitory concentration, NOR: Norbornene,

PEG: Polyethylene glycol, INH: Isoniazid, ACM: Absolute concentration method

Table 2: DST for the resistant strain using luciferase reporter MPs

\begin{tabular}{|c|c|c|c|c|}
\hline \multirow[t]{2}{*}{ Strains } & \multicolumn{2}{|c|}{ Solvent control } & \multicolumn{2}{|c|}{ INH $(0.2 \mu \mathrm{g} / \mathrm{ml})$} \\
\hline & Control 1 & Control 2 & Plain INH & NOR+PEG+INH \\
\hline $\begin{array}{l}\text { Resistant } \\
\text { strain }\end{array}$ & 1269 RLU & 750 RLU & 760 RLU (24\%) & 625 RLU (37\%) \\
\hline
\end{tabular}

DST: Drug susceptibility testing, INH: Isoniazid, NOR: Norbornene,

PEG: Polyethylene glycol, RLU: Reduction in light units,

MPs: Mycobacteriophages

work [18-20]. Emission of light in a sample infected with phages and supplemented with luciferin indicates the presence of viable bacterial cells [21]. In our study, viable cells were less compared to drug-free control; this proves that the compound is active.

LRP assay results shows that INH along with NOR has a better antimycobacterial activity compared to plain INH which is shown in Table 2 and proved that anti-TB drugs along with nanocarrier are stable under extracellular conditions. Strains exhibiting a reduction of less than $50 \%$ relative light units in the drug containing vial compared to control were classified as resistant. The resistant strain was inhibited by NOR+PEG+INH at a rate of $37 \%$ compared to plain INH which had a inhibition rate of $24 \%$ thus by further standardizing the inhibitory activity of NOR+PEG+INH can be increased at a higher rate.

Cytotoxicity assay of anti-TB drugs done in IISER proved that at lower concentrations of the drug, the cytotoxicity could not attribute to damage to cytoplasmic, endosomal and lysosomal membranes, and normal human cell death can be prevented when exposed to NOR [11]. The nanodrug conjugates have high drug loading efficiency up to 58$95 \%$, so that more amounts of drugs which is proposed to act on target site can be loaded [22]. In vivo activity using Thp1 cell line for the promising drug delivery is in process.

\section{CONCLUSION}

The antimycobacterial susceptibility testing of nanocarrier with antiTB drugs revealed that NOR-based INH with $74 \%$ of INH has lower MIC value. The predominant anti-TB drug INH plays a major role in treatment of TB infection both in intensive and continuous phase. Thus, enhancing activity of INH with NOR conjugates appreciated as a welcoming step. The nanosized polymers with anti-TB drugs are expected on future treatment for TB infection.

\section{ACKNOWLEDGMENTS}

The authors are grateful to acknowledge SRM University, Kanchipuram for the facilities to carry out the research, IISER Kolkata for providing the nanocarrier with drugs for the study and NIRT for providing TB strains and valuable suggestions toward the study.

\section{REFERENCES}

1 Fivy K, Syed AS, Syed WG. Study on drug-resistant tuberculosis and tuberculosis treatment on patients with drug resistant tuberculosis in chest clinic outpatient department. Int $\mathrm{J}$ Pharm Pharm Sci 2012;4:733-7.

2. Dutta PK, Das JK. An overview of information, education and communication (IEC) activities in revised national tuberculosis control programme (RNTCP) and role of NGOs. Indian J Prev Soc Med 2012;43(4):366-71.

3. Amit D, Dharmeshkumar P. A profile of adverse effects of antitubercular drugs. GCSMC J Med Sci 2016;1:37-41.

4. Schaberg T, Rebhan K, Lode H. Risk factors for side-effects of isoniazid, rifampin and pyrazinamide in patients hospitalized for pulmonary tuberculosis. Eur Respir J 1996;9(10):2026-30.

5. Orme IM Development of new vaccines and drugs for TB: Limitations and potential strategic errors. Future Microbiol 2011;6(2):161-77.

6. Dye C, Espinal MA. Will tuberculosis become resistant to all antibiotics? Proc Biol Sci 2001;268(1462):45-52

7. SosnikA, CarcabosoAM, Glisoni RJ, Moretton MA, Chiappetta DA. New old challenges in tuberculosis: Potentially effective nanotechnologies in drug delivery. Adv Drug Deliv Rev 2010;62(4-5):547-59.

8. Ruckmani K, Kumaresan C. Sustained release aerosol for pulmonary drug delivery system: A review. Int J Pharm Pharm Sci 2013;5:126-30.

9. Pandey R, Khuller GK. Subcutaneous nanoparticle-based antitubercular chemotherapy in an experimental model. J Antimicrob Chemother 2004;54(1):266-8.

10. Mane SR, Dinda H, Sathyan A, Das Sarma J, Shunmugam R. Increased bioavailability of rifampicin from stimuli-responsive smart nano carrier. ACS Appl Mater Interfaces 2014;6(19):16895-902.

11. Vijayakameshwara RN, Shivshankar RM, Abhinoy K, Jayasri DS, Raja S. Norbornene derived doxorubicin copolymers as drug carrires with $\mathrm{pH}$ responsive hydrazone linker. Biomacromolecules 2012;13:221-30

12. Shivshankar RM, Vijayakameswara RN, Raja S. Reversible pH and lipid sensitive vesicles from amphiphilic norbornene-derived thiobarbiturate homopolymers. ACS Macrolett 2011;1:482-8.

13. Shivshankar RM, Koushik C, Himari D, Jayasri DS, Raja S. Stimuli responsive nanocarrier for an effective delivery of multifrontline tuberculosis drugs. Polym Chem 2014;5:2725-35.

14. NIRT Manual. Standard Protocols for Mycobacteriology Laboratory, Version 1.1, November; 2010.

15. Azger D, Vanaja K, Narayana PR. Constructiona and evaluation of luciferase reporter phages for detection of active and non-replicating tubercle bacilli. J Microbiol Methods 2009;7(31):18-25.

16. Sarvamangala D, Nagasejitha P, Phrabu S, Srinivas L, Murthy US. Preparation and evaluation of isoniazid nano-conjugates for improving the therapeutic efficiency. Int J Pharm Sci Res 2015;6:739-45.

17. Jacob WR, Bartella RG, Udani R, Kalkut T, Sarkis GJ, Hatfull GF, et al. Rapid assessment of drug susceptibility testing of Mycobacterium tuberculosis by means of luciferase reporter phage. Science 1993;260:819-22.

18. Sarkis GJ, Jacobs WR Jr, Hatfull GF. L5 luciferase reporter mycobacteriophages: A sensitive tool for the detection and assay of live mycobacteria. Mol Microbiol 1995;15(6):1055-67.

19. Schlossberg D. Tuberculosis: A Sourcebook for Nursing Practice. New York: Springer Publications; 1995.

20. Banaiee N, Bobadilla V, Osornio JS, Luciferase reporter mycobacteriophages for detection, identification and antibiotic susceptibility testing of MTB in Mexico. J Clin Microbiol 2001;39:3883-8.

21. Suarez S, O'Hara P, Kazantseva M, Newcomer CE, Hopfer R, McMurray DN, et al. Airways delivery of rifampicin microparticles for the treatment of tuberculosis. J Antimicrob Chemother 2001;48(3):431-4.

22. Sharma A, Sharma S, Khuller GK. Lectin-functionalized poly (lactide-co-glycolide) nanoparticles as oral/aerosolized antitubercular drug carriers for treatment of tuberculosis. J Antimicrob Chemother 2004;54(4):761-6. 